\title{
La herencia indoeuropea en la religión griega: Arquíloco, Calímaco y Estrabón
}

Jordi REDONDO*

\section{La base indoeuropea de la religión griega arcaica}

El problema del origen indoeuropeo de la religión griega parece desde algun tiempo zanjado sobre la base de un status quo según el cual dicha herencia no habría sido tan determinante como la recibida del Oriente Próximo; en su conjunto, las creencias orientales y mediterráneas habrían configurado el ideario griego en mayor medida que las derivadas del legado indoeuropeo. Burkert, por ejemplo, no deja lugar a dudas cuando afirma lo siguiente: (...) For Dumézil as well as for Alföldi the specifically Greek material is particularly unproductive: here Greek culture seems more indebted to Neolithic-Anatolian urban culture than to Indo-European nomads ${ }^{ }$.

Del lado de los investigadores que más han hecho por situar la religión de la Grecia antigua en el seno de la herencia común a los pueblos indoeuropeos, un nombre tan señero como el de Georges Dumézil ha llegado a reconocer la particularidad de la posición griega. Se trataría de una manifestación tan sólo menor, sometida a modificaciones de gran calado, de las que la más significativa sería, tal vez, la falta de definición precisa de los dioses pertenecientes a las funciones segunda y tercera ${ }^{2}$. Para Dumézil,

\footnotetext{
* Universitat de València - jordi.redondo@uv.es - Agradecemos sinceramente las sugerencias que se nos han hecho llegar, y que nos han permitido fijar mejor nuestra posición respecto a determinados problemas de los textos citados.

I W. Burkert, Greek Religion, Oxford, 1985, p. I8. Burkert se refiere a A. Alföldi, Die Struktur des voretruskischen Römerstaates, Heidelberg, 1974. Para Dumézil, véase la nota siguiente.

2 G. Dumézil, L'idéologie tripartie des Indo-Européens, Bruxelles, 1958, p. 91: La Grèce-par rançon sans doute du 'miracle grec', et aussi parce que les plus anciennes civilisations de la mer Égée ont trop forte-
} 
estaríamos ante una mera herencia literaria, sin mayor reflejo en la estructura e ideología de la sociedad griega ${ }^{3}$.

En este estado de cosas, parece que quienes defienden la plena participación de la cultura religiosa de la antigua Grecia en la herencia indoeuropea se ven abocados a aportar las oportunas pruebas, como si el conjunto de los datos hasta ahora disponibles hablara de manera contundente en favor de la tesis opuesta. En otras palabras, la afirmación del carácter genuinamente indoeuropeo de la religión griega se vería muy comprometida por la abundante existencia de manifestaciones diversas -en el panteón, en el culto, en los mitos- sin correlación alguna con las demás religiones de los pueblos indoeuropeos.

La realidad de la actividad investigadora demuestra, sin embargo, lo contrario. Hay un cierto numero de trabajos específicamente dedicados a poner de relieve el carácter indoeuropeo de la religión griega en sus diversos aspectos. Destaca a este respecto la obra de Bernard Sergent, quien ha recogido ejemplos de la pervivencia de numerosos cultos y esquemas sociales de inequívoca matriz trifuncional en Esparta, Jonia, Beocia y Creta, además de ponderar el papel del santuario délfico como centro difusor de un sistema religioso igualmente basado en la trifuncionalidad ${ }^{4}$. No obstante, la conclusión

ment marqué les envahisseurs venus du Nord-contribue peu à l'étude comparative: même les traits les plus considérables de l'héritage y ont été profondément modifiés. B. Sergent, «L'utilisation de la trifonctionnalité d'origine indo-européenne chez les auteurs grecs classiques», Arethusa 13, 1980, p. 233-278, 233: On connaît le paradoxe présenté par la Grèce dans le domaine de ses origines culturelles: alors que sa langue est typiquement indo-européenne, plus conservatrice même que plusieurs autres de la même famille pour nombre de traits, l'essentiel de la mythologie et de la symbolique indo-européenne, telles qu'elles ont puêtre reconstituées par comparaison des données indiennes, iraniennes, latines, scandinaves, ne se retrouve chez elle qu'en très faible partie, et / ou amplement modifié. Para una crítica de la teoria dumézialiana, véase C. Scott Littleton, «Towards a Genetic Model for the Analysis of Ideology: The Indo-European Case», Western Folklore 26, 1967, p. 37-47; M.V. García Quintela, Georges Dumézil (I898-1986), Madrid, 2006 (reimpr.) (= Dumézil. Une Introduction, Crozon, 2006).

3 G. Dumézil, op. cit., p. 58-59: En Grèce, où l'essentiel de la religion n'est sûrement pas indo-européen, le groupement des déesses dans la légende du berger Pâris, par exemple, reste un jeu littéraire, ne forme évidemment pas une authentique combinaison religieuse.

4 B. Sergent -ademas del trabajo ya citado en n. 2-, "La représentation spartiate de la royauté», RHR 189, 1976, p. 3-52; «Le partage du Péloponnèse entre les Héraklides», $R H R$ 190, 1977, p. I21-136; «Les trois fonctions des Indo-Européens dans la Grèce ancienne: Bilan critique», Annales ESC, 1979, p. II55-1186; y Les trois fonctions indoeuropéennes en Grèce ancienne. De Mycènes aux tragiques, París, 
de Sergent parece a primera vista un tanto sorprendente: los reflejos del principal rasgo caracterizador de la religión indoeuropea, la trifuncionalidad, no habrían pasado de una presencia modesta en la Grecia arcaica, y sólo a partir de la obra de Platón habrían recuperado una cierta influencia en la cultura helénica, si bien tan sólo en el plano intelectual y por un breve plazo de tiempos.

Todavía en un plano preferentemente literario y filológico, a los trabajos de Sergent hay que añadir los del propio Dumézil ${ }^{6}$, los de Allen, que ha establecido las relaciones entre numerosos pasajes de la literatura griega arcaica y la literatura y la mitología indias ${ }^{7}$, los de Cuartero y Pàmias a propósito del análisis de la propia tradición

1998. Sobre cultos y ritos de carácter trifuncional en Esparta, Tebas y Creta, vease «L'utilisation»... p. 250, 250-25I, 253 y 255-256, respectivamente. Para el caso específico de Esparta, «La représentation»... y «Le partage»... Sobre el papel de Delfos, "Les trois fonctions"... p. II-I2. Más allá del estudio específico de la ideología trifuncional, el estudio de la herencia indoeuropea en la cultura griega se plasma en otras tres importantes monografías de Sergent, L'homosexualité dans la mythologie grecque, París, 1984 , L'homosexualité initiatique dans l'Europe ancienne, París 1986, y Homosexualité et initiation chez les peuples indoeuropéens, París, 1996, además de una cuarta en que se ocupa específicamente del caso griego, Les trois fonctions indo-européennes en Grèce ancienne, París, 1996.

5 B. Sergent, «L'utilisation»..., ibid.: Les thèmes trifonctionnels sont bien rares dans les poèmes homériques, ils jouent un rôle par contre important chez Hésiode, mais c'est avec Platon, au IV s., qu'ils occupent, un moment, le devant de la scène, et donnent lieu aux plus magnifiques développements. Sólo la supervivencia de la ideología trifuncional en ámbitos particulièrement conservateurs habría permitido que des auteurs d'époques relativement tardives aient pu en être informés et les avoir utilisés avec profit. Sobre la trifuncionalidad en Platón, véase G. Dumézil, Mythe et Épopée I. L'idéologie des trois fonctions dans les épopées des peuples indo-européens, París, 1968, p. 493-496.

6 G. Dumézil, «Les trois fonctions dans quelques traditions grecques», in L'éventail de l'histoire vivante. Hommage à Lucien Febvre II, París, 1953, p. 25-32, cf. 28-32 sobre Hdt. I 30-33, 34-45 y 86-92, a propósito de las leyendas de Cleobis y Bitón y Creso.

7 N.J. Allen, "Arjuna and Odysseus: a comparative approach", SALG Newsletter 40, 1993, p. 39-43; "Why did Odysseus become a horse?", JASO 26, 1997, p. I19-I3I; “The hero's five relationships: a ProtoIndo-European story", in J. Leslie (ed.), Myth and Myth-making, Londres, 1996, p. I-20; "Homer 's simile, Vyasa's story", Journal of Mediterranean Studies 6, 1996, p. 206-211; "The Indo-European Prehistory of Yoga", International Journal of Hindu Studies 2, 1998, p. I-20; "Arjuna and the second function: a Dumezilian crux", Journal of Royal Asiatic Society 9, 1999, p. 403-418; "Les Crocodiles qui se transforment en nymphes", Ollodagos 14, 1999, p. I5I-167; "Argus and Hanu man: Odysseus 's dog in the light of Mahâbhârata", JIES 28, 2000, p. 3-16; "Imra, Pentads and Catastrophes”, Ollodagos 14, 1999, p. 278-308; "Athena and Durgâ: warrior goddesses in Greek and Sanskrit epic", in S. Deacy and A. Willing (edd.), Athena in the Classical World, Leiden 2001, p.367-382; "Pénélope et Draupadî: la validité d'une compa- 
mitográfica griega ${ }^{8}$ y el de Pralon sobre la filosofía del siglo IV a.C. ${ }^{9}$ Nosotros mismos nos hemos ocupado ya de esta cuestión en una ocasión anterior, al tratar de las figuras trágicas de Orestes y Pílades ${ }^{\mathrm{I}}$. En un plano diferente y sobremanera necesario, que otorga una atención principal a los datos arqueológicos, hay que citar la contribución de Yoshida sobre un conjunto de la relevancia del templo de Apolo en Delfos ${ }^{\text {II }}$.

Como una de las razones principales del desequilibrio existente entre la religión griega y el resto de religiones indoeuropeas se ha aducido por parte de otro investigador francés, Pierre Levêque, la aportación derivada de la invasión doria, o, por expresarnos en términos más actuales, del asentamiento de los dorios en el Peloponeso ${ }^{12}$. De forma por completo paradójica, la aportación de los dorios no habría supuesto el reforzamiento de los vínculos de la cultura griega con la tradición indoeuropea, sino, justo al contrario, una acentuación del perfil mixto de la religión griega, definida por la fusión de las creencias indoeuropeas con otras de procedencia tanto oriental como mediterránea. Nótese que la teoría de Levêque choca, precisamente, con la observación de Sergent respecto a la mayor vigencia de los esquemas trifuncionales en la sociedad lacedemonia. Además, el recurso metodológico a la invasión doria parece un expediente de muy dudosa fiabilidad.

En nuestra opinión, y en consonancia con una línea interpretativa bien definida, muchos fenómenos tanto ideológicos como sociales de la Grecia clásica no sólo derivan

raison", La Mythologie et l'Odyssée, Hommage à Gabriel Germain, Ginebra, 2002; "Mahâbhârata and Iliad: a common origin?", Annals of the B.O.R.I. LXXXIII, 2002, p. 165-175.

8 F. Cuartero, «Hèracles, fundador de sacrificis: l'heroi i les tres funcions», Faventia 20, 1998, p. 15-25; J. Pàmias, «Dumézil, el perro y la jabalina. Un mito de transferencia», REA I०8, 2006, p. 483-492.

9 D. Pralon, «Le modèle triparti dans la philosophie grecque du IVe siècle a.C.», in J. Bonnet \& D. Pralon (edd.), Georges Dumézil. Cahiers pour un temps, París, 1981, p. I21-136.

Io J. Redondo, «Pílades, doble d'Orestes, o l'emancipació d'un personatge”, in K. Andresen, J.V. Bañuls \& F. De Martino (edd.), La dualitat en el teatre, Bari, 2000, p. 37I-39r.

II A. Yoshida, «Le fronton occidental du temple d'Apollon à Delphes et les trois fonctions», $R B P h H$ 44, 1966, p. 5-II, cf. 7: Il est à noter, dans ces conditions, que cette triade reproduit la structure du système trifonctionnel des Indo-Européens etc. Del mismo autor, véase también un trabajo anterior, "Sur quelques coupes de la fable grecque”, REA 68, 1965, p. 31-36, sobre la versión délfica de la leyenda de los Siete Sabios de Grecia.

I2 P. Levêque, "I dori e la religione delle età buie", in D. Musti (ed.), Le origini dei Greci. Dori e mondo egeo, Roma \& Bari, 1990, p. 259-276, 271-272. 
a todas luces de la herencia trifuncional, sino que además detentan una posición central en la estructura de las respectivas sociedades. Si tomamos como ejemplo de revisión de algunos de los postulados establecidos por Dumézil el de la infrarrepresentación de la segunda función, que lo estaría tan sólo por las figuras de una diosa, Atenea, y de Ares $^{13}$, convendría hacer algunas precisiones: en primer lugar, la figura de Atenea no se ha de encuadrar sin más ni más en el género femenino, por de pronto por las especiales características de su nacimiento, que hacen de ella un prototipo de antifémina, y luego porque la feminidad misma de las divinidades requiere un análisis detenido, como ya demostró Nicole Loraux ${ }^{\mathrm{I}}$; en segundo lugar, la proximidad de las funciones primera y segunda permite que dioses de la primera representen ambas, como es el caso de Zeus, debelador de gigantes y titanes ${ }^{15}$; en tercer lugar, la época arcaica griega no fue testimonio de grandes contiendas bélicas, sino de conflictos a escala reducida, como los enfrentamientos en la costa tracia, por lo que no habría que esperar una preeminencia en ella de los dioses de la segunda función, más bien al contrario; en cuarto lugar, la propia mitología indoeuropea no es una construcción cartesiana, sino que reproduce las inquietudes de una determinada sociedad; en el caso de la religión griega, la herencia indoeuropea se entiende bien sin la necesidad de alegar para cualquier aparente disfunción la influencia determinante de una cultura religiosa mediterránea u oriental. La aludida infrarrepresentación, en fin, no compromete la viabilidad y operatividad del esquema trifuncional, como demuestran los usos literarios e iconográficos, con total

I3 Así lo afirma el propio P. Levêque, op. cit., p. 272: In Grecia, la funzione guerriera resta schiacciata, $e$ d'altronde è eteroclita, poiché è in parte nelle mani di una dea, Atena. En realidad, si lo que se pretende es cuestionar la representación de la segunda función, debe explicarse la integración en el panteón de Ares, un dios ambiguo, importado de Oriente y denostado por el propio Homero. En su figura pueden reconocerse, por ejemplo, el mitema indoeuropeo del guerrero que es hallado culpable de sacrilegio respecto a las tres funciones, cf. U. Strutynski, «Ares: A Reflex of the Indo-European War God?», Arethusa I3, 1980, p. 217-231, a la vez que notables diferencias que lo separan de sus correlatos en otras culturas indouropeas.

I 4 N. Loraux, «Che cos'è una dea?”, in P. Schmitt Pantel (ed.), Storia delle donne. I. L'Antichità (= Histoire des femmes en Occident I. L'Antiquité, París, 1991, (= Bari 1997), p. 13-55.

is Cf. C. Scott Littleton, The New Comparative Mythology: An Anthropological Assessment of the Theories of Georges Dumézil, Berkeley 1982 ${ }^{3}$, p. 219-220: It is possible, perhaps, to suggest that the classic definition of Zeus (i.e., that of Hesiod) dates from a time when the Greeks had ceased to think of terms of a clear distinction between the first and the second function. 
independencia del número de dioses que hayamos de encuadrar en tal o cual función. Por otra parte, la teórica sobrerrepresentación de la tercera función ha sido explicada mediante el recurso a una herencia cretominoica ${ }^{16}$, cuando para muchos de los cultos y ritos relativos a la fertilidad puede hallarse una clara ascendencia indoeuropea.

Sendos ejemplos de pervivencia de elementos religiosos indoeuropeos nos permitirán replantear la cuestión de dicha herencia tanto en la época arcaica, en la que se data la obra del yambógrafo Arquíloco, como en un contexto cronológico diferente del habitual: las épocas helenística e imperial, en las que se sitúan las respectivas obras de dos autores de muy diferente género, el también poeta Calímaco y el geógrafo e historiador Estrabón.

\section{Las tres funciones en un fragmento de Arquíloco}

En la exigua obra del yambógrafo Arquíloco de Paros, el fragmento is West ${ }^{17}$, que debemos a una cita de Plutarco ${ }^{18}$, dice lo siguiente ${ }^{19}$ :

No me importan los bienes de Giges, rico en oro, ni menos aún me ha invadido jamás el ansia de emulación, ni soy tampoco celoso de las gestas de los dioses, y tampoco estimo el imponente poder del tirano; lejos están de mis ojos.

A nadie se le escapa que las tres ambiciones rechazadas por la persona loquens -el obrero Carón, según el testimonio de Aristóteles, del que Plutarco precisa que era de Tasos-, son la riqueza, el honor y el poder. Se corresponden a la perfección con las funciones tercera, segunda y primera, respectivamente. Así lo indican las alusiones a las riquezas del rey del país más próspero en el entorno de la Grecia del siglo VII a.C., Lidia, cuna de la moneda y paradigma de opulencia; a las $\theta \epsilon \hat{\omega} v$ ép $\rho \alpha$, que evicentemente no puede referirse a labores fabriles, agrarias, domésticas o similares, ni a empresas amorosas, sino que ép $\gamma$ ov significa hazaña, gesta, con preferencia aquella conseguida

I6 P. Lévêque, op. cit., ibid.: La terza funzione è sovrabbondantemente rappresentata: lo era a cominciare dal sincretismo cretese-miceneo, el'introduzione di nuovi dèi asiatici-un dio-ragazzo e una Grande Deanon ha fatto che rafforzarla.

I7 M.L. West, Iambi et elegi Graeci ante Alexandrum cantati I, Oxford, $1989^{2}$.

I8 Plut. De tranquillitate animi io, 470b. Una alusión al pasaje, que incluye la cita del primer verso, se encuentra ya en Aristóteles, Retórica III 17, $1418 b_{23}$.

19 Las traducciones son del autor. 
en el campo de batalla, razón por la cual se denomina ép $\rho \alpha$ a $v \theta \rho \omega ́ \pi \omega \nu$ a las labores referidas a la producción de bienes ${ }^{20}$, de manera complementaria y por oposición a estas $\theta \epsilon \hat{\omega} v$ Łép $\gamma \alpha$; y, por fin, a la máxima expresión del poder personal, la tiranía, que completa la serie.

La secuencia escogida por Arquíloco difícilmente puede ser debida a la casualidad. El bueno de Carón hace profesión de una postura ideológica que hoy encuadraríamos entre las propuestas llamadas antisistema. El rechazo expresado por Arquíloco coincide con otros textos del mismo autor, en los que da réplica al legado cultural y literario de la épica, como el fragmento il 4 West, en el que se decanta por un modelo guerrero que es la antítesis del consagrado por Homero. La exigüidad del pasaje impide reconocer en él con claridad lo que en nuestra opinión es un reflejo de la ideología de la sociedad griega de la época. Podrá objetarse, pues, que se trata de un tópico literario, sin relación alguna con el pensamiento religioso contemporáneo. Creemos, sin embargo, que el texto de Arquíloco se enmarca en un preciso horizonte ideológico que se atiene al esquema trifuncional, como acontece, por ejemplo, en la descripción del escudo de Aquiles, a menos que ésta responda también al perfil de un nuevo tópico.

\section{Un mito indoeuropeo en Calímaco}

El interés del poeta helenístico Calímaco por los temas religiosos no ha escapado a la atención de los críticos modernos. En general, se pondera en él su respeto por la tradición mítica, lo que, unido a su tendencia a tratar de temas anticuarios, su magnífica formación académica y la disponibilidad de medios de la Biblioteca alejandrina, lo convierte en una fuente a tener muy en cuenta en materia de religión y mitología ${ }^{21}$. El primer pasaje que vamos a comentar es el siguiente:

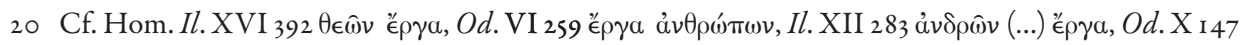

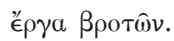

2I Sobre el tratamiento de los temas religiosos y mitológicos en la poesía de Calímaco, véase A.W. Bulloch, «The Future of a Hellenistic Illusion. Some observations on Callimachus and religion», $M H_{41}$, 1984, p. 209-230; G. Zanker, Alexandrian Poetry and Realism. A Study on Literature and Audience, Sydney/Londres, I985, p. II 4; M.W. Haslam, "Callimachus' Hymns", in M.A. Harder, R.F. Regtuit \& G.C. Wakker (eds.), Callimachus, Groningen 1993, p. III-I25. 
Ella, copiosa en vientos e inamovible, unica entre las islas, batida por las olas tiene en el mar hundidas sus raices, más practicable para las gaviotas que para los corceles; aquel que inmenso gira en torno suyo enjuga en ella la infinita espuma de la mar Icaria; por eso la han poblado los arponeros que las aguas surcan. Pero no está sujeto a aprensión en su contra el que se cuente entre las principales islas, cuando junto al Océano y Tetis, hija de los Titanes, se congregan, y va en todo momento a la cabeza de las demás; tras de sus buellas sigue luego la fenicia Cirno, en nada despreciable, y la extensa Abantíade, la de los helopieos, Sardo, de encanto llena, y aquella cuya orilla ganó primero Cipride desde la mar adentro, y a cuenta de ese arribo la protege. Ellas, sí, están fortificadas por baluartes que en derredor brindan seguro abrigo, mas Delos por Apolo: ¿qué muro más impenetrable habria? Rocas y murallas serian abatidas bajo el impulso del estrimonio Bóreas; pero un dios es siempre inasequible a todo daño. Tal valedor, amada Delos, te ha ofrecido su amparo.

Si innumerables cantos te rodean, ¿con cuál te engarzaré? ¿Qué ansiarias oir? ¿Acaso cómo el poderoso dios, que al principio los montes hería con el arma de triple filo que le forjaron los Telquines, creaba con su esfuerzo las islas que en la mar están, y a todas las alzó de raiz y las hizo rodar hacia la mar, y en lo hondo de su abismo las plantó por su base, para que se olvidaran de la tierra firme? Pero no te apremió la obligación, sino que a tu albedrio surcabas los mares, y tenias por nombre el antiguo de Asteria porque saltaste a una profunda fosa desde el cielo, cuando escapabas al enlace con Zeus, semejante a una estrella: por aquel tiempo aún no te frecuentaba la áurea Leto, aún por aquel entonces eras Asteria y no te llamabas Delos. Marineros que iban desde Trecén, ciudad del Janto, en dirección a Éfira, te avistaron muchisimas veces en aguas del golfo Sarónico, pero al regresar de Éfira ya no te vieron por segunda vez, sino que te pusiste a correr por el rápido cauce del angosto Euripo, que entre el fragor discurre, y el mismo día, tan pronto como bubiste desdeñado las aguas de la mar calcídica, nadaste en pos del cabo Sunio, de dueños atenienses, o hacia Quios, o hacia la eminencia, bañada enteramente por las olas de la isla Partenia -pues aún no era Samos-, en la que te hospedaron las ninfas micalésides, las vecinas de Anceo. Mas cuando a Apolo ofreciste tu solarpara su nacimiento, te impusieron 
las gentes de la mar un diferente nombre porque ya no surcabas invisible las aguas, sino que tú en las olas del Egeo bincaste firmemente tu asiento ${ }^{22}$.

Nuestra intención es la de centrarnos en un aspecto del mito, el que describe la isla de Asteria. Desde nuestro punto de vista, Calímaco está retomando aquí un viejo mito indoeuropeo que ha hallado su mayor difusión en la cultura céltica, y que ha sido reconocido como tal por Sergent ${ }^{23}$. Se trata del motivo de la isla errante, oculta por continuas brumas, un tema que ha causado extrañeza entre los estudiosos de la mitología griega ${ }^{24}$. La crítica moderna de Calímaco ya ha reconocido en este Himno diversas innovaciones en lo que respecta al tratamiento de la materia mítica, uno de los cuales sería, precisamente, el de la aparición del personaje de la ninfa Asteria ${ }^{25}$. Sin embargo, esta figura mítica era ya conocida, como lo demuestra un pasaje de Píndaro: poblaron también dispersas islas de abundante fruto y obtuvieron la muy gloriosa Delos, ya que Apolo les concedió habitar el solar de Asteria ${ }^{26}$. Asteria/Delos tiene, por tanto, naturaleza divina, y al acoger a la olímpica Latona parece prenunciar el sincretismo de las dos figuras/islas. Así lo indica el cambio por el nombre de Delos del antiguo de Asteria, cuya etimología, pace Sergent, se ha de poner en relación con el carácter origi-

22 Call. Del. vv. 28-54. Trad. J. Redondo, Calimaco. Himnos. Epigramas, Madrid 1996.

23 La amabilidad de M.V. García Quintela ha puesto en nuestro conocimiento el artículo de B. Sergent, «Une Dèlos celtique», Ollodagos 19, 2005, p. I17-138, que examina los mismos pasajes de Calímaco y llega a las mismas conclusiones que nosotros. El pasaje principal de Calímaco, Del. 28-54, en la traducción de P. Villalba, Callímac. Himnes, Barcelona, Fundació Bernat Metge, 1972, ha sido objeto de un comentario pormenorizado según las líneas maestras de nuestra interpretación desde el curso 1996-1997. Más adelante trataremos de los aspectos en que el presente análisis difiere del de Sergent.

24 C. Penglase, Greek Myths and Mesopotamia. Parallels and Influence in the Homeric Hymns and Hesiod, Londres \& Nueva York 1994, p. 243: Many ideas appear to be background knowledge and are referred to only in passing, never to be explained. Ideas such as these are the dark wave which is driven by shrill winds against Delos as the birth of Apollo, and the idea of the floating island itself, for which a completely satisfying reason is never given.

25 Cf. W.H. Mineur, Callimachus' Hymn to Delos. Introduction and Commentary, Leiden 1984, p. 75 : Another new element in Callimachus'version of the myth is probably the origin of Delos as the nymph Asteria etc.

26 Pi. Pae. 5, 38-43. 
nario de isla errante, y no con el que más tarde identifica a la isla, y que está, en cambio, expresado mediante la nueva denominación ${ }^{27}$.

El mito celta no era desconocido de la literatura y la cultura griegas. Hay una exposición bastante completa en el testimonio que nos ofrece Posidonio, en cita de Estrabón:

Dice <sc. Posidonio> que en el océano hay una pequeña isla no del todo en alta mar, que se halla ante la desembocadura del río Lígero <el moderno Loira> ; y que la habitan las mujeres de los namnetes ${ }^{28}$, que están poseidas por Dioniso y aplacan a este dios por medio de ritos y otras prácticas sagradas desusadas; y que en dicha isla no pone el pie varón alguno, sino que haciéndose a la mar ${ }^{29}$ las mujeres mismas tienen trato con varones y se vuelven de regreso; y que es costumbre que una vez al año deshacen y de nuevo rehacen la techumbre del templo en un mismo día, antes de la puesta de sol, aportando cada una un capazo; y aquella a la que se le caiga el capazo, es descuartizada por las demás; y que

27 La etimología de Asteria podría encerrar una formación sobre el adjetivo $\sigma \tau \epsilon \rho \in$ ós -compárese el verbo

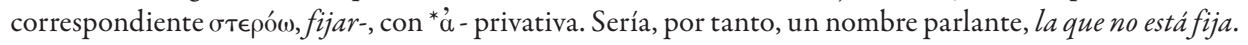
B. Sergent, «Une Dèlos celtique»... p. 130, acepta la calidad de nomen parlans del topónimo Asteria,

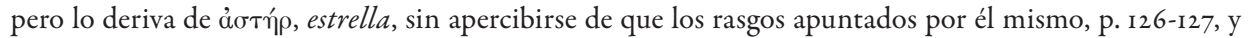
confirmados por el testimonio de Píndaro, frgs. 78-79 ed. Snell \& Mähler, indican claramente que la isla deja de ser errante cuando Latona llega a ella y surgen de las entrañas de la tierra cuatro columnas de bronces que la sostienen y fijan; a la vez, las brumas desaparecen para hacer que la isla pase a ser luminosa y bien visible, con lo que el antiguo nombre de Asteria es reemplazado por el de Delos. Veamos el texto de Píndaro: Salve, obra de los dioses, retoño deliciosísimo para gozo de los hijos de Latona, de esplendorosas trenzas, hija de la mar, prodigio inamovible de la vasta tierra, que los mortales llaman Delos, y estrella que de lejos se avista, desde la azulada tierra, en el Olimpo, los bienaventurados dioses. Estaba antaño sujeta al trasiego, a merced de las olas y de las acometidas de toda suerte de vientos; pero cuando en ella hizo pie la del linaje de Ceo entre acelerados dolores de parto, cercanos al alumbramiento, al punto entonces cuatro columnas erguidas se alzaron desde las raices de la tierra, y asentadas en cimientos de incorruptible acero, sujetaron con sus infulas la roca, donde ella al dar a luz contempló su feliz progenie. Cuando Sergent, ibid., razona que Dèlos s'appela d'abord Astéria, nom tiré du nom de 'l'étoile' astêr -une étoile est un point lumineux-et surtout le nom même de Dèlos ne signifie pas autre chose que 'la Visible' etc., pasa por alto que la transformación de la isla exigía esta redenominación. Si ambos nombres significaran lo mismo, el cambio del antiguo por el moderno perdería todo su sentido.

28 El texto de Estrabón presenta la lectura $\Sigma \alpha \mu \nu \iota \tau \hat{\omega} v$, carente de sentido, y que Tyrwhitt enmendó con

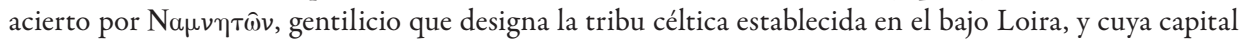
coincide con la actual Nantes.

29 El participio $\pi \lambda \varepsilon 0 v \alpha \sigma \alpha \varsigma$ indica que en este caso son las mujeres las que navegan, mientras la isla permanece inmóvil. Este aspecto del mito no ha quedado recogido por la leyenda que recoge Posidonio. 
en tanto portan sus despojos en torno al templo entre gritos de 'evohé' no cesan hasta que ponen fin a su rabia; y que sucede siempre que caiga alguna, que ha de padecer dicha suerte. (...) Cuenta todo ello en términos más bien propios de los mitos, pero más dignos de crédito respecto a Deméter y Core, cuando dice que hay una isla frente a Bretaña en la que se llevan a término prácticas sagradas semejantes a las que hay en Samotracia en relación con Deméter y Core. También es noticia de las que merecen crédito la siguiente, que en el pais celta florece un árbol semejante a la higuera, y que produce un fruto parecido a un capitel corintio; y cuando se le hace una incisión vierte un jugo que untado en flechas es mortal 30 .

El texto de Estrabón no recoge todas las características del mito, pero sí las suficientes para identificarlo. Destacaremos la existencia en la(s) isla(s) de prácticas sagradas antiguas y cruentas llevadas a cabo por mujeres. Si bien el motivo se encuentra profusamente atestiguado en el área céltica ${ }^{31}$, se ha recogido también su presencia en el folclore

30 Strab. IV 4, 6. Para la relación del pasaje con la religión celta en la península Ibérica, véase M.V. García Quintela, «El reyezuelo, el cuervo y el dios céltico Lug: aspectos del dossier ibérico», Arys 5, 2002, p. 153-202, p. 166-179.

3I Cf. T.D. Kendrick, The Druids. A Study in Keltic Prehistory, Londres, 1966 (= 1927), p. 138-139: These women, described by Mela, were possessed of marvellous magical powers, and might be approached to those who set sail especially to consult them. There was also another community of women, devoted to orgiastic cults, established on an island near the mouth of the Loire whereon no man was allowed to set foot. Estrabón podría referirse a la isla de Yeu, rica en monumentos megalíticos y que ofrece además una explicación geológica para el tema de las islas errantes, ya que según las épocas ha estado unida al continente o bien se ha separado de él. Por otra parte, hay que destacar que Yeu no era una isla poblada regularmente, sino un santuario al que se acudía en determinadas épocas del año. La geografía, sin embargo, sugiere otras tres islas, conocidas en francés como Belle-Île, Hoëdic y Houat, y en la lengua del país, en bretón, Enez an Gerveur, Edig y Enez Houad, respectivamente. Belle-İle puede ser descartada por haber sido habitada durante la época antigua, pero no así las otras dos, de menor extensión, que fueron pobladas en época prehistórica al estar unidas a tierra firme, y deshabitadas luego al transformarse en islas. Todas ellas muestran vestigios arqueológicos de gran riqueza. Véase también C. Squire, Celtic Myth \& Legend, Poetry \& Romance, Nueva York 1979, p. 394: Pembrokeshire -the ancient Dyfed-has kept the tradition most completely. The story goes that there is a certain square yard in the hundred of Cemmes in that count which holds the secret of the fairy realm. If a man happens to set his feet on it by chance, his eyes are opened, and he can see that which is hidden from other men -the fairy country and commonwealth-, but, the moment he moves from the enchanted spot, he loses the vision, and he can never find the same place again. That country is upon the sea, and not far from shore; it may sometimes be sighted by sailors. Véase, en fin, S. O'Sullivan, Folktales of Ireland, Chicago, UP 1966, p. 274: There are many traditional stories of islands that appear and vanish off the Irish Coast as well as of sunken town. Permítasenos señalar, a este respecto, el 
de la Grecia moderna ${ }^{32}$. El tratamiento en Calímaco es por completo coherente con los rasgos del mitema: la isla puede personalizarse en la figura de una mujer; se desplaza allende la mar con velocidad inusitada; los marineros la avistan, pero no pueden alcanzarla ni mucho menos tomar tierra en ella; está cubierta por una espesa bruma, aunque puede también aparecer de improviso a los ojos de los mortales; y guarda relación con los mitos escatológicos, al estar concebida como una morada para los amados de los dioses $^{33}$.

Procedamos partiendo de aquello que conocemos bien. En primer lugar, la identificación de la isla de Delos con el mundo de la mar está fuera de toda duda. Así lo atestiguan, por ejemplo, los numerosísimos grafitos de temas marinos presentes no sólo en la antigua zona portuaria, sino por toda la isla ${ }^{34}$. Ahora bien, no existe un vínculo

fondo folclórico de la novela de Miguel de Unamuno San Manuel Bueno, mártir, a propósito de la ciudad sepultada en el fondo de un lago. Para este motivo de la ciudad anegada por las aguas en la mitología y el folclore celtas, véase J. Rhys, "Triumphs of the Water-World”, Celtic Folklore, Welsh and Manx I, Oxford I9OI, p. 40I-455, 417-4I8.

32 S. Thompson, Motif-Index of Folk-Literature. A Classification of Narrative Elements in Folktales, Ballads, Myths, Fables, Mediaeval Romances, Exempla, Fabliaux, Jest-Books, and Local Legends (Bloomington, Indiana University Press, 1966, III p. 203-204, señala hasta siete matrices de relatos que incluyen el motivo en cuestión, que identifica con la matriz F737. Las otras seis son Ai79.2, con el motivo de la isla flotante otorgada en señorío a un héroe como don de una divinidad; A955.I2, con el motivo de la anciana que ostenta la custodia de una isla flotante propiedad de los dioses; Di524.4, con el motivo de la isla que se transforma en bote, y viceversa, y que está relacionado con el motivo D936; Di643.3, con el motivo de la isla que se traslada a voluntad de su dueño, también en relación con D936; D2136.6, con el motivo de la isla que se desplaza por arte de magia, igualmente en relación con D936; y, por fin, F388.2, con el motivo de las hadas que se reúnen en una isla flotante. En la descripción del motivo F737, Thompson, que lo caracteriza porque la isla se desplaza según su voluntad y aparece y desaparece a la vista, sitúa sus registros en el área celta y en Islandia, además de Grecia y Hawai. Evidentemente, esta última localización no se relaciona con una herencia indoeuropea, pero en cambio no resulta novedoso que el folclore griego conserve leyendas antiquísimas. La tradición griega se sitúa en Eolia, cf. W.S. Fox, The Myths and Legends of All Races I. Greece and Rome, Boston, 1916, p. 266.

33 F. Nansen, In Northern Mists II, Londres 1911, p. 150: (...) The Promised Land in Irish Myth is surrounded by darkness (= fog) like the Norwegian huldrelands and the Icelandic elflands. En época altomedieval el mito enlaza con el ciclo artúrico, con la leyenda de Avalon. En la tradición griega, este aspecto se halla en la Isla Blanca o Isla de los Bienaventurados a la que Tetis traslada el cadáver de su hijo Aquiles. Para una interpretación alternativa, cf. G. Alford, "Elysion. A Foreign Eschatological Concept in Homer's Odyssey", JIES I9, I991, p. I5I-I6I.

34 Cf. L. Basch, «Graffites navals à Dèlos», BCH Supp. I. Études Déliennes, París, 1973, p. 65-76, 66: (...) Loin d'être cantonnés dans un secteur de l'île, les graffites navals sont omniprésents: quartier du théâtre, 
entre la figura del dios Apolo y el mundo marino. Los datos históricos, arqueológicos y literarios coinciden en señalar como responsables de esa identificación de la isla con la mar a otras figuras religiosas, en una cronología que se extiende hasta las culturas minoica y micénica; así lo prueban los cultos a Glauco y Brizo ${ }^{35}$. Del primero pueden apreciarse algunos rasgos en la figura de Poseidón, con el que habría sincretizado: así lo sugieren la protección que ambos brindan a marineros y pescadores, el carácter oracular y la relación con sacrificios cruentos. La segunda, de la que por desgracia sólo tenemos referencias literarias, se reconoce como una divinidad marina y a la vez oracular ${ }^{36}$.

Hay otro mitema heredado del que dar cuenta, y en el que Calímaco se extiende con cierto agrado:

Y entonces, los cisnes servidores del dios que entona el canto describieron un circulo, tras de dejar atrás el Pactolo meonio, hasta por siete veces en derredor de Delos, y con sus melodias el nacimiento embelesaron las aves de las Musas, aves las más canoras de las que el aire surcan; de abi que el niño atara a la lira, más tarde, tantas cuerdas cual veces cantaron los cisnes, durante los dolores de parto de su madre. Ya no cantaron una octava vez, pues él surgió a la luz; y las ninfas Delíades, de un rio antigua estirpe, por largo tiempo el canto sagrado de Ilitía entonaron, y al punto hizo sonar el broncíneo firma-

du stade, îlot des Comédiens, maisons aux stucs, de l'Hermès, de l'Inopos, du lac.

35 L. Basch, op. cit., p. 70: L'île d'Apollon a honoré en effet de très bonne heure un nombre exceptionnel de divinités protectrices de la navigation: Poseidon, Amphitrite, les Néréides et Glaucos y ont eu des attaches qui pourraient remonter au stade pré-apollinien des cultes de Dèlos. Glaucos, qui y rendait des oracles, était peut-être une survivance préhellénique. À ce panthéon marin vint s'ajouter, à l'époque hellénistique, une nouvelle divinité, l'Isis Pelagia, patronne de la navigation. Mais la plus antique des divinités marines honorées à Délos fut vraisemblablement Brizô, qui n'était pas exempte d'affinités avec la Britomartis-Dictynna crétoise. Cette déesse, dont le culte n'est attesté qu'à Dèlos, agréait des offrandes faites dans des vases en forme

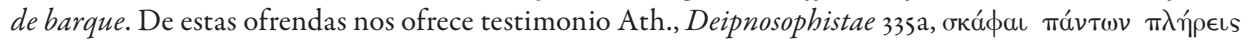

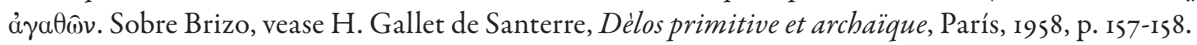

36 P. Bruneau, Recherches dans les cultes de Dèlos à l'époque hellénistique et à l'époque impériale, París, 1970, p. 447: Le culte de Brizô n'est attesté que par des textes littéraires, ou, plus exactement, par le seul Sémos, car Eustathe résume Sémos et les notices d'Hésychius et de l'Etymologycum Magnum paraissent en découler aussi. Se refiere al testimonio de Semo en Ateneo, Deipnosophistae 335 a b; fuentes secundarias son Eustacio en su comentario a Hom. Od. XII 252, más las voces B Magnum. Sobre el doble carácter, marino y a la vez oracular, véase el mismo P. Bruneau, op. cit., p. 448. 
mento penetrante clamor, mas no se llenó Hera de un afán de venganza porque de ella arrancó Zeus toda cólera ${ }^{37}$.

El motivo de los siete círculos que los cisnes trazan en torno a la isla de Delos justo antes del nacimiento de Apolo y Ártemis tiene un paralelo en el nacimiento del dios Heimdall, cuyo corte indoeuropeo no impide que fuera también acogido entre las leyendas de época cristiana. En el caso de Heimdall, son nueve olas las que consecutivamente preceden su nacimiento ${ }^{38}$. Al tratarse de una divinidad marina ${ }^{39}$, tenemos la impresión de que este motivo se ha insertado entre los mitos relativos a Apolo tan sólo de una manera secundaria, ya que en origen se habría relacionado con alguna de las divinidades protectoras de la isla de Delos, probablemente Glauco.

Ayuda a conformar el marco mitológico en que se mueve Calímaco un nuevo elemento del que tenemos también pruebas firmes. Hacia el final del Himno se recuerdan prácticas de iniciación de carácter cruento:

Asteria de incontables altares, de innumerables preces, ¿qué marino en el curso de sus giras por el Egeo ha pasado de largo junto a ti en su rauda nave? No soplan sobre ella unos tan recios vientos ni la obligación fuerza una cuanto más pronta travesia; al contrario, se pliega el ligero velamen, y no embarca de nuevo sin que antes dé una vuelta a tu altar golpeándose ${ }^{40}$ al par de los azotes, y muerda con las manos a la espalda el sagrado

37 Call. Del., vv. 249-259, trad. J. Redondo, op. cit.

38 H. D’Arbois de Jubainville, Cours de littérature celtique II. Le cycle mythologique irlandais et la mythologie celtique, París i884, p. 156-157.

39 G. Dumézil, Comparative Remarks on the Scandinavian God Heimdall», in E. Haugen (ed.), Gods of the Ancient Northmen, Berkeley, 1973 (= "Remarques comparatives sur le dieu scandinave Heimdall", Études Celtiques 8, 1959, p. 263-283), p. 126-140, I26 sobre el carácter del dios, 132-138 sobre el motivo exacto de su nacimiento en los confines de la mar.

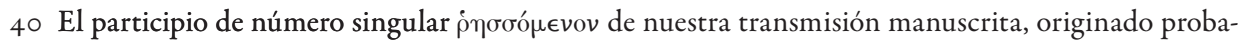
blemente en la mala lectura de una abreviatura, y que mantienen las ediciones de Pfeiffer, Cahen, Villalba y D’Alessio, fue oportunamente corregido por Meineke en $\dot{\eta} \gamma \sigma \sigma o \mu$ évous, enmienda que obedece a una restauración textual mínima. W.H. Mineur, op. cit., p. 248, defiende el texto de los códices apoyándose en Homero, Iliada XVIII 57 y y H.Ap. 516, lo que le hace concluir que middle or passive forms of the verb are not attested elsewhere. Sin embargo, la realidad es que desde la época clásica se documentan usos medio-pasivos de verbos que en Homero son sólo activos. Véase nuestro trabajo "Non-epic features in the language of Apollonius Rhodius", in M.A. Harder, R.F. Regtuit \& G.C. Wakker (edd.), Apollonius

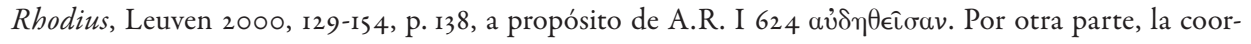


tocón de un olivo; juegos éstos que una ninfa de Delos inventó como solaz risueño del aún niño Apolo ${ }^{41}$.

La arqueología ha desvelado la presencia en Delos de prácticas cruentas, aunque a la hipótesis de un carácter religioso se añaden otras dos: la del castigo a unos enemigos condenados en el curso de una guerra, o la pena infligida a unos criminales ${ }^{42}$. Sin embargo, sabemos por Tucídides que los atenienses al menos tenían por impuro el derramamiento de sangre en la isla de Apolo ${ }^{43}$. Destacan, por un lado, el hecho de que la purificación de época de Pisístrato comprendiera todo aquel área que se pudiera abarcar a la vista desde el templo; por otro, la prohibición, datada en la segunda purificación, tanto de morir en la isla como de dar a luz. Es decir, al estar la isla consagrada a la divinidad no debía haber en ella signo alguno de carácter mortal; al haber sido Delos solar del parto de Latona, tampoco mujer alguna podía parir en la isla, sin olvidar tampoco que el parto implica también derramamiento de sangre, considerada impura. Por consiguiente, desde finales de la época arcaica al menos, ya que la ordenación dictada por Pisístrato apunta a la codificación de una práctica consuetudinaria, ritual, y no a la creación de una norma, la tradición religiosa vetaba la aplicación en Delos de la pena capital ${ }^{44}$. Por otra parte, la decapitación no figura jamás entre las

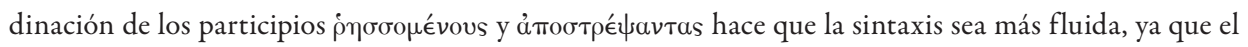
contexto narrativo no favorece la inconcinnitas.

4 I Call. Del. vv. 316-324, trad. J. Redondo, op. cit.

42 P. Ducrey \& N. Ducrey, «Les suppliciés de Fourni», BCH Supp. I. Études Déliennes, París, 1973, p. 172-I8I. El hallazgo consiste en un esqueleto casi completo más parte de la pierna de un segundo esqueleto. Ambos cadáveres muestran que las muertes se produjeron a consecuencia de sendas torturas, como atestiguan las señales de clavos en brazos y piernas. No habrían sido crucificados, sino fijados a una plancha o tabla. El esqueleto más completo, y este rasgo ha de destacarse, fue decapitado, probablemente para acelerar la muerte, piensan los autores del trabajo, que se inclinan por el castigo a unos criminales, tal vez piratas.

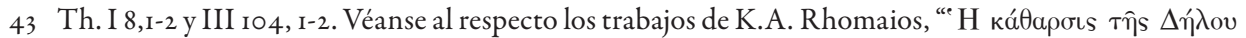

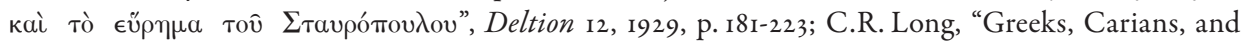
the Purification of Delos", AJA 62, 1958, p. 297-306; y S. Hornblower, "The Religious Dimension to the Peloponnesian War, or, What Thucydides Does Not Tell Us”, HSClPh 94, 1992, p. 169-197.

44 B. Sergent, «Une Dèlos celtique»..., p. 132 y 136, sugiere que este aspecto del ciclo mítico délico habría sido introducido por los atenienses al tiempo de la fundación de la Liga Deloática. A ellos se debería, pues, la transformación de la isla en un lieu strictement religieux (p. 136), lo que habrían hecho mediante la aplicación de un mito indoeuropeo. En nuestra opinión, tanto el carácter religioso de la isla 
prácticas propias de una sentencia, sino que tiene una función de índole religiosa, reservada tan sólo, en epoca histórica, al escarnio del enemigo abatido en guerra. El hecho de que los ajusticiados de la isla de Delos fueran decapitados nos hace pensar en un ritual de carácter religioso, sin duda anterior al establecimiento del culto apolíneo. Calímaco habría trasladado al Himno, en su particular visión de la arqueología mítica de la isla, el recuerdo de estas prácticas cruentas, si bien transmutado en una suerte de raí propios de los cuidados para con un recién nacido.

Repasemos los mitemas presentes en el texto de Calímaco: en primer lugar, una divinidad femenina que acoge a una diosa en el contexto de la hora del parto; luego, una isla que se desplaza allende la mar y que se hace invisible a voluntad; unas aves que unen sus armoniosos cantos de celebración a los de un coro de doncellas; y finalmente unos cultos sangrientos. Veamos ahora otro elemento de interés en lo que respecta a las tradiciones religiosas delias recogidas por el poeta, en esta ocasión tomado del Himno a Apolo:

Con cuatro años, la primera vez, trabó unos fundamentos Febo en Ortigia la bella, cabe a la orilla de una marisma circundada de tierra firme. Artemis, que se hallaba de caza, sin cesar trajinaba cabezas de cabras cintiades, de modo que Apolo tejía su altar: sobre de cuernos construyó su asiento, con cuernos ensambló el altar, córneos muros, en fin, en derredor alzaba. Asi aprendió Febo a levantar los primeros basamentos ${ }^{45}$.

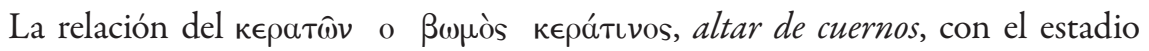
que convencionalmente denominamos religión micénica está atestiguada por materiales arqueológicos abundantes y diversos ${ }^{46}$. La singularidad de este altar le valió en la Antigüedad el nombre de altar de Delos, que es como lo denomina el lexicógrafo Pólux ${ }^{47}$.

como su vinculación con el mito de la isla errante han de retrotraerse al segundo milenio a.C., si bien el primero sería ya de origen preindoeuropeo.

45 Call. Apoll., vv. 58-64, trad. J. Redondo, op. cit.

46 M.P. Nilsson, “The Horns of Consecration and the Façade of the Minoan Shrines", The MinoanMycenaean Religion and Its Survival in Greek Religion, Lund, 1950², p. 165-193. Véase también, en otra línea de interpretación, C. Miralles, "L’arc i la lira. Aproximació a la lectura de l'Himne II de Callímac", in Athlon. Satura grammatica in honorem F.R. Adrados II, Madrid 1987, p. 636-639.

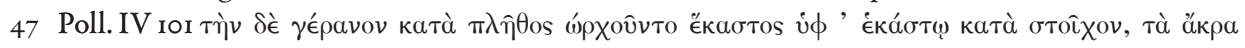

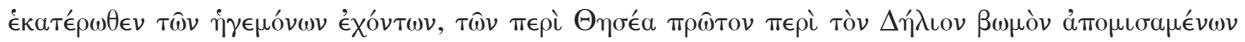


De los cuatro motivos que hemos enunciado más arriba sólo el segundo, el de la isla errante, tenía un origen indoeuropeo, de acuerdo con nuestra investigación. Es mérito de Sergent el de haber demostrado el nexo existente entre la isla de Delos y la mítica Emain del Viaje de Bran, definida ésta última también por el triple carácter de la fecundidad, del delicioso canto de aves y doncellas y de la paz ${ }^{48}$.

Cerraremos este repaso al Himno a Delos de Calímaco con la continuación del pasaje precedente, en el que se hace alusión a otros importantes cultos delios:

Asteria, la de intenso olor a incienso, en derredor de ti formaron un círculo las islas y como en torno a un coro te ciñeron; sedeño de cabello. Héspero no te avista callada ni silente, sino en todo momento circundada de voces. Salmodian los unos el canto del venerable licio, el canto que en tu honor trajo el adivino Olén desde orillas del Janto; ellas, las danzarinas, el firme suelo baten con su pie. Entonces es por fin colmada de coronas la tan nombrada sacra efigie de la antigua Cípride, la que otrora Teseo en unión de sus hijos erigiera, cuando volvia de Creta; ellos, huidos del salvaje vástago de Pasifae y su penoso mugido, y de la curva sede del sinuoso laberinto, ob soberana, en torno de tu altar en círculo danzaron asi que iba templándose la cítara, y fue Teseo jefe de ese coro. Desde ese dia envian los Cecrópidas, cual sempiterna ofrenda a Febo, los cordajes de aquella nave, hoy portadora de una pía embajada ${ }^{49}$.

No interesan a nuestro propósito las referencias al ciclo de Teseo, salvo en la medida en que confirman la presencia en el poema de contenidos religiosos y mitológicos muy antiguos, próximos a la época micénica o propios de ella. Así lo indican el encuentro de Teseo y Ariadna, que habría tenido lugar en Delos, y que vuelve a enlazar prácticas cultuales continuadas hasta el fin de la Antigüedad con los mitos del segundo milenio a.C. La danza de la grulla, $\gamma$ épavos, ha sido perfectamente interpretada por Sergent a la luz de una sección del poema céltico del Viaje de Bran, con lo que este elemento del mito redunda en la impronta indoeuropea de gran parte del material délico ${ }^{50}$. Se trata, por tanto, de una quinta coincidencia entre el mito griego y el celta,

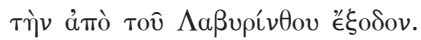

48 B. Sergent, «Une Dèlos celtique»... p. I28, I30-131 y 133.

49 Call. Del., vv. 300-315, trad. J. Redondo, op. cit.

50 B. Sergent, «Une Dèlos celtique»... p. 134-135. 
todos ellos heredados de la tradición común. Ahora bien, merece un comentario detenido la alusión al canto de una composición del poeta Olén de Sardes, y por tanto en lengua licia, ya que coincide con el testimonio del Himno homérico a Apolo, vv. 156-164: Junto a todo ello hay un gran portento cuya fama jamás se extinguirá, las doncellas delias, servidoras de quien de lejos hiere con susflechas; asi que ellas por de pronto han entonado el himno en honor de Apolo, y a su vez de Latona y de Artemis, cantan un himno en recuerdo de varones y mujeres de tiempos antiguos, y embelesan las razas de los hombres. De todas gentes saben imitar hablas y jerigonza. Cada cual diría que él mismo estaba hablando, tan bien compuesto es su hermoso canto ${ }^{51}$.

Tras este texto se trasluce la existencia de una doble práctica cultual en el santuario de Delos que incluiría himnos en honor del dios en dos lenguas, tanto la griega como una lengua diferente, probablemente anatolia ${ }^{52}$. La lengua griega utilizada en los himnos debía contener arcaísmos susceptibles de ser interpretados como rasgos característicos de los diferentes dialectos. En cuanto a la expresión alóglota, el término que se registra en el Himno homérico a Apolo, $\beta a \mu \beta a \lambda \iota a \sigma \tau u ́ s$, no incide ni en el desprecio ni en la ridiculización, pero el tono entre afectivo y familiar lo sitúa en un contexto de escasa formalización, y próximo en cambio a un ámbito de cotidianeidad. Lo que nos interesa subrayar es cómo, una vez más, Calímaco se hace eco de creencias y cultos que remontan a un pasado lejano.

La conclusión a que llegamos, de acuerdo con la interpretación que acabamos de presentar, muestra cómo para la composición del Himno a Delos-como del Himno a Apolo- el autor tuvo en cuenta una serie de tradiciones de gran antigüedad. Probablemente Calímaco combinó las leyendas transmitidas oralmente sobre la isla de Delos con fuentes literarias de carácter religioso, al estilo de los Himnos homéricos que pretendía superar, y que no han llegado hasta nosotros. Unas y otras fuentes comparti-

si H. Ap., vv. 156-164.

52 La hipótesis de la coexistencia de dos lenguas, una de ellas es la griega, fue formulada en primer lugar por U. von Wilamowitz-Möllendorf, Die Ilias und Homer, Berlín 1916, p. 450-451. Para interpretaciones alternativas, véase C. Brillante, «La voce che affascina: Elena e Cleopatra», contribución a un coloquio celebrado en Rávena en el año 2004 y disponible en la dirección electrónica siguiente, http://www. dismec.unibo.it/musichegreci/web2004/brillante2004.htm. 
rían una temática diversa, reflejada por el poeta de uno u otro modo, pero con el comun denominador de un origen remoto.

\section{La descripción y elogio de Europa en Estrabón.}

El siguiente pasaje de que vamos a ocuparnos está tomado del geógrafo Estrabón, que al presentar el continente europeo hace del mismo una descripción de carácter programático, que es a la vez un elogio de sus gentes y de su organización política. Por motivos textuales en esta ocasión debemos remitirnos en primer lugar al original:

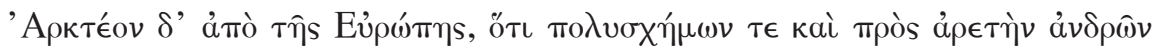

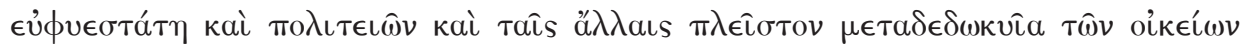

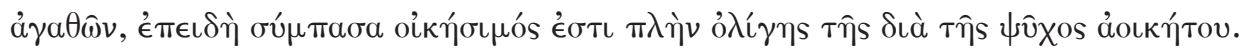

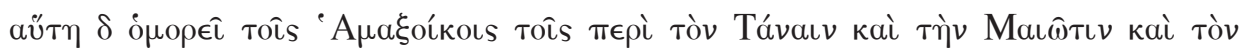

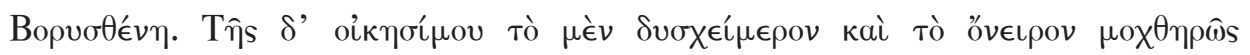

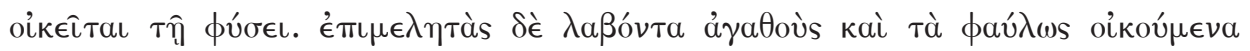

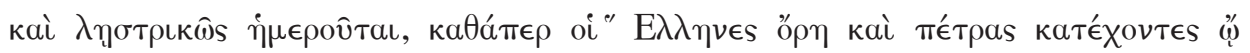

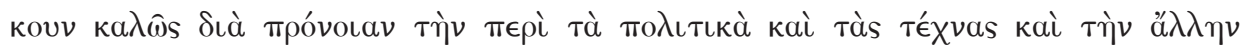

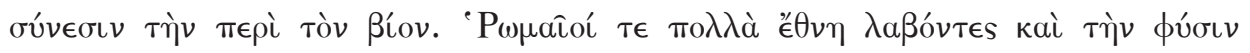

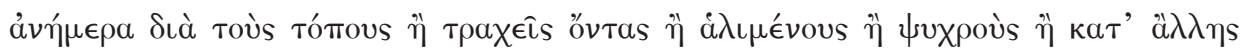

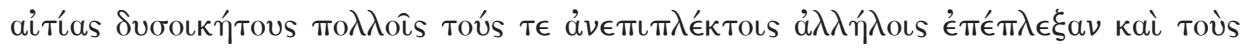

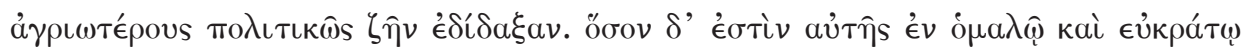

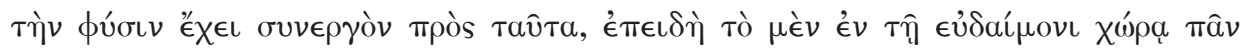

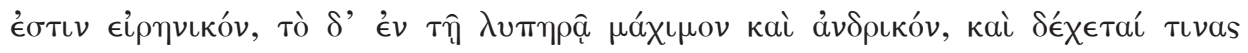

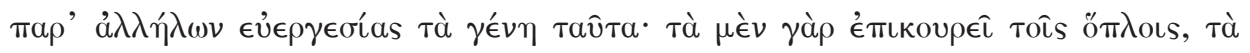

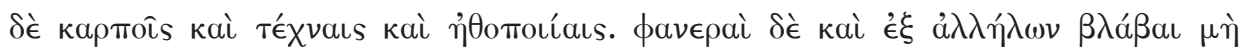

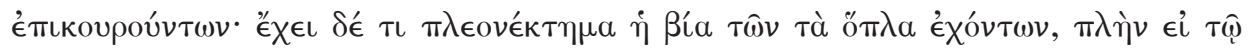

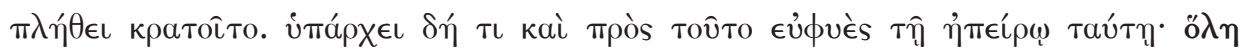

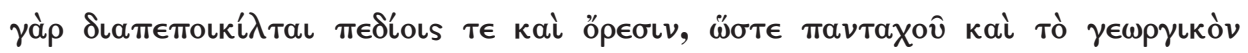

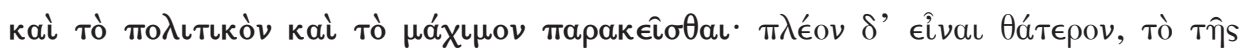

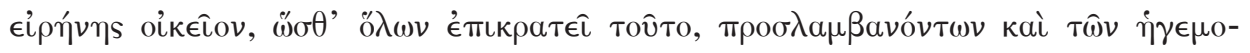

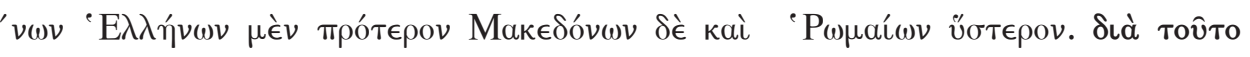

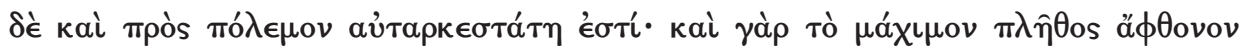




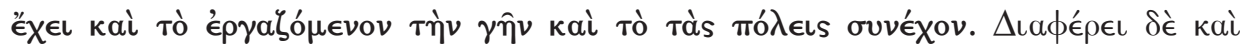

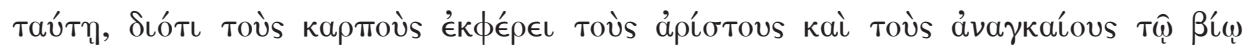

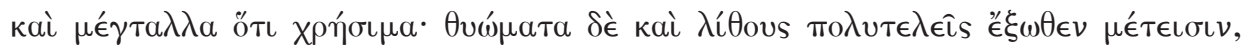

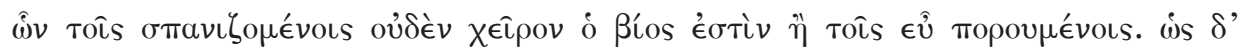

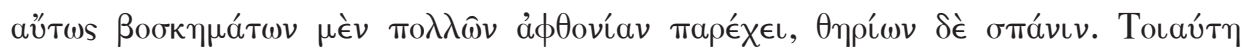

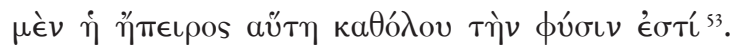

Hemos señalado en negrita las frases que interesan a nuestro propósito, aun cuando su perfecta comprensión sólo puede darse si atendemos al significado de todo el pasaje. Para la fijación del texto suele adoptarse la enmienda de Pletón $\gamma \in \omega \rho \gamma \iota \kappa o ́ v ~ \tau \epsilon$,

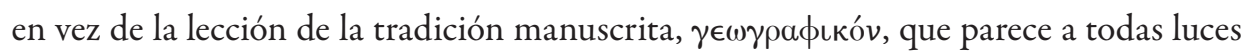
un error antiguo y que de ninguna manera casa con el sentido del pasaje. En nuestra

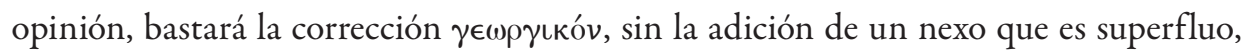
además de restar claridad y fuerza al polisíndeton kaí. Hay que señala también la

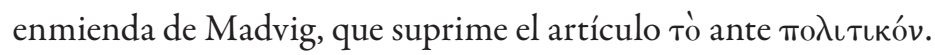

Leamos en primer lugar el pasaje en la edición y traducción inglesa de Jones, que

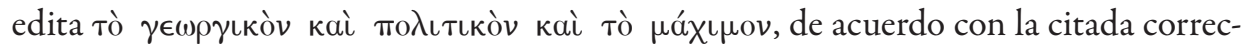
ción de Madvig: However, this continent has a natural advantage to meet this condition also; for the whole of it is diversified with plains and mountains, so that throughout its entire extent the agricultural and civilised element dwells side by side with the warlike element; but of the two elements the one that is peace-loving is more numerous and therefore keeps control over the whole body ${ }^{54}$.

Al prescindir tanto del triple nexo кaí como del triple artículo determinado tò, Jones destruye la secuencia ternaria del pasaje de Estrabón, con lo que el sentido del texto queda del todo desvirtuado. Lo mismo ocurre con la más reciente edición y traducción publicada, la francesa de Germaine Aujac. Su traducción dice así: Or il se trouve que, sous ce rapport aussi, notre continent est naturellement bien doué, car il est entièrement composé d'une mosaïque de plaines et de montagnes, de sorte que partout

53 Strab. II 5, 26.

54 H.L. Jones, The Geography of Strabo I, Cambridge, I960, p. 487. 
coexistent la tendance paysanne et sociale et l'instinct guerrier. C'est le premier élément qui domine, celui qui porte à la paixss.

Ambas traducciones, la inglesa y la francesa, se centran en una oposición entre dos tipos de sociedad, tendentes, respectivamente, a preservar la paz y a preparar la guerra, y así lo entiende Aujac en su comentario al pasaje ${ }^{56}$. Esta interpretación es antigua, pues la enmienda de Madvig va en esa misma línea, y se apoya en la frase siguiente, $\pi \lambda \lambda_{\text {éov } \delta}$ '

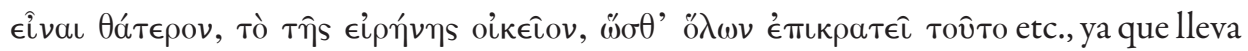
a entender que se trata efectivamente de sólo dos elementos contrapuestos. Así sería de tratarse de un texto de época clásica, pero no es el caso de Estrabón, que integra en su lexis numerosos rasgos sintácticos propios de la koiné. Entre éstos figura una innovación en el empleo del pronombre contrastivo ét $\epsilon$ pos, que puede referirse a más de un antecedente, y lo mismo sucede con á $\mu \phi o ́ \tau \epsilon \rho s^{57}$. Luego puede entenderse que, para Estrabón, dos de ellos tienen la preeminencia, cuanto es propio de la paz, de suerte que prevalecen sobre el conjunto.

55 G. Aujac, Strabon. Geographie II, París, 1969, 109-1 Io.

56 Cf. G. Aujac, op. cit., I66, n. 3: Si l'on adopte le texte de Pléthon, les autres manuscrits étant immanquablement fautifs en cet endroit, on fait ressortir l'opposition entre les tendances pacifiques (agriculture et vie organisée en société) et les tendances guerrières. Le même lien entre agriculture et administration se retrouve quelques lignes plus loin, opposé aussi a la tendance belliqueuse. Tampoco hay la menor observacion en G. Aujac, Strabon et la science de son temps, París, 1966, y «Strabon et son temps», in W. Hübner (ed.), Geographie und verwandte Wissenschaften, Stuttgart 2000, p. 103-139. Lo mismo cabe decir de E.H. Warmington, Greek Geography, Nueva York, 1973 (= Londres \& Toronto, 1934), p. 213.

57 A.T. Robertson, A Grammar of the New Testament in the Light of Historical Research, Nashville 1934, p. 749: But $\boldsymbol{\epsilon}^{\prime \prime} \boldsymbol{\tau} \boldsymbol{\rho} \rho$ os comes also to be employed merely for 'another' with more than two and with no idea of difference. (...) We are not surprised then to learn that the papyri furnish plenty of examples where $\epsilon^{\prime \prime} \tau \epsilon \rho$ os refers to more than two. Así lo recogen también W.F. Arndt \& F.W. Gingrich, A Greek-English Lexicon of the New Testament, Chicago, University Press, $1952^{4}$, p. 315, s.u. $\epsilon^{\prime \prime} \tau \epsilon \rho$ os, apartado b), que lleva el explícito epígrafe de of more than two. Para ilustrar cuán extendido estaba este nuevo uso tanto de

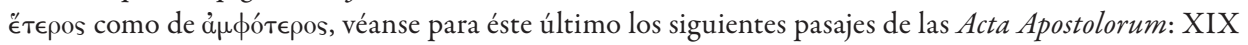

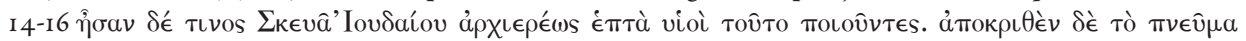

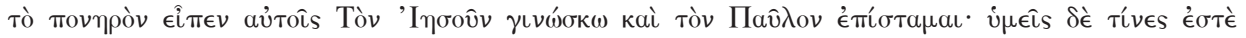

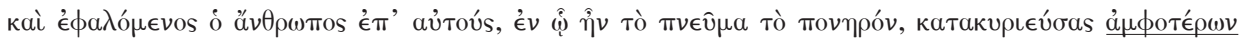

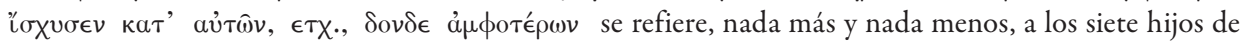

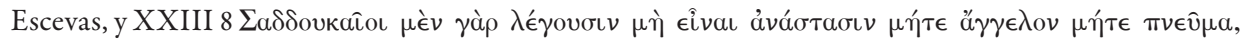

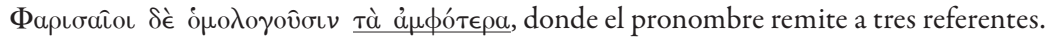


Obsérvese que nuestro geógrafo hace explícita por dos veces la expresión de una división del continente en tres tipos de áreas equitativamente distribuidas a lo largo de todo su territorio, división que Aujac refleja perfectamente en su traducción: Tant pour la paix que pour la guerre, l'Europe est totalement autonome: elle posséde une réserve inépuisable d'hommes pour se battre, pour travailler la terre et pour administrer les cités (subrayado nuestro) ${ }^{58}$. Se recogen así las tres clases de ciudadanos, soldados, campesinos y gente de ciudad, representativas, respectivamente, de las funciones segunda, tercera y primera. Al tratarse de una doble cita, nuestra interpretación se ve harto reforzada. Por otra parte, la idea que en nuestra opinión refleja el pasaje estraboniano se inscribe en una tradición que Bernard Sergent ha estudiado en autores de géneros tan dispares como el lírico Tirteo, la ciencia médica del Corpus Hippocraticum y los filósofos Platón y Aristóteles ${ }^{59}$. La conclusión de Sergent subraya cómo los diversos pasajes muestran la aplicación de la trifuncionalidad a una idea muy precisa del mundo, del que Grecia -y, mejor aún, Atenas, de acuerdo con Jenofonte ${ }^{60}$ es el centro mismo, y por tanto le corresponden los beneficios y ventajas del equilibrio, la justa belleza y la supremacía. En suma, aquello que los griegos conocían como кó $\mu$ os, el orden sancionado por los dioses.

Es conocida, en la Geografía de Estrabón, la interrelación de geografía y organización política. El medio físico no es sino el reflejo de una sociedad y de una forma de gobierno $^{6}$. Pero hay a la vez una dimensión mitica que no hemos de soslayar, y que la sintaxis del pasaje ayuda a reconstruir. Por un lado, conviene recordar que Estrabón era

58 G. Aujac, op. cit., II 5, 26.

59 B. Sergent, «Ethnopsychologie et climats en Grèce ancienne», in E. Katz, A. Lammel \& M. Goloubinoff (edd.), Entre ciel e terre. Climat et sociétés, París, 2002, p. 25-38. Los pasajes analizados son Tyrt. frg. 9 ed. Diehl, C.H. Aër. 12-23, Pl. Re. 435d-436a, y Arist. Pol. 1327 a-b. Sobre el pasaje hipocrático -que tiene un exacto paralelo en Hdt. IX I2I, como recuerda el propio Sergent, op. cit. p. 37 i n. 24-, véase el detallado estudio de J.A. López Férez, «'Sobre los aires, aguas y lugares', capítulo i6: estaciones del año, instituciones políticas y modo de ser de los asiáticos», in J.A. López Férez (ed.), Estudios actuales sobre textos griegos, Madrid, I991, p. 175-203.

60 B. Sergent, «Ethnopsychologie»..., p. 37.

6 I Cf. C. Nicolet, Geography and Politics in the Early Roman Empire, University of Michigan Press, 1991 (= L'Inventaire du Monde: géographie et politique aux origines de l'Empire Romain, París, 1988; trad. it., Roma \& Bari, 1989), p. 73: (...) Geography is indeed the science of the appropiation of the land by man, the inventory of his home, of his resources, and of the traces that he had left behind him. This geography 
un científico de formación ecléctica, en la que destacan tanto el empirismo de matriz aristotélica como el legado de la escuela estoica, en especial a través de Posidonio ${ }^{62}$. Por otro, que en su obra hay un profundo y amplio reconocimiento del papel de la poesía, aspecto en el que coincide con el historiador Polibio ${ }^{63}$. La reivindicación de Homero, por ejemplo, se produce ya desde el proemio de la Geografía, donde -justo al revés que Tucídides- Estrabón presenta al autor de Ilíada y Odisea como el primer geógrafo digno de ese nombre ${ }^{64}$. Y no se trata tan sólo de Homero, ya que la Geografía muestra un repertorio ciertamente amplio de citas de poetas. Ahora bien, más importante aún es la función de estas citas, que enlaza directamente con el discurso antropológico ${ }^{65}$. Más aún, comienza a entreverse el empleo no sólo de citas tomadas de la épica, el drama y la lírica, sino también de materiales mitográficos diversos, entre los cuales hay que citar a Apolodoro y Hecateo ${ }^{66}$.

is strictly political; it is essentially aimed at the rulers in order to allow them to govern better; but it also determines the types of government.

62 Cf. C. Horst Roseman, "Reflections of philosophy: Strabo and geographical sources", in D. Dueck, H. Lindsay \& S. Pothecary (edd.), Strabo’s Cultural Geography. The Makingof a Kolossourgia, Cambridge, University Press, 2005, p. 27-4I, p. 29: (...) Stoic concentration on physics and the natural sciences certainly attracted him, and perhaps their rigour and dogma did as well. P. 39: The philosophic perspective Strabo brought to his work on geography was an eclectic blend of his early Aristotelian training and his commitment to the general tenets of the Old Stoa. Sobre la influencia de Posidonio, especialmente en los libros I y II, cf. 30-3I.

63 Cf. A.M. Biraschi, "Strabo and Homer: a chapter in cultural history", in D. Dueck, H. Lindsay \& S. Pothecary (edd.), op. cit., p. 73-85, p. 75, a propósito de la crítica moderna: Strabo's ties with Stoic tradition were underlined and also his relationship with Polybius who, as can be seen in the Prolegomena, himseld fefended the 'historical truth' of Homeric information against Eratosthenes.

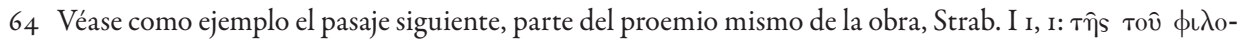

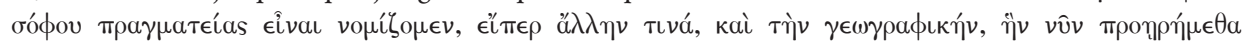

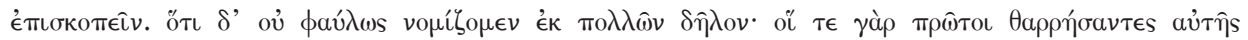

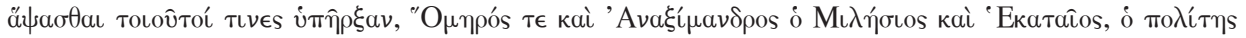

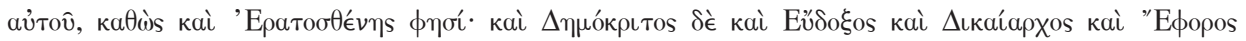

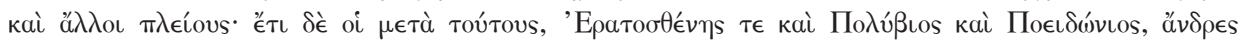
$\phi \iota \lambda$ ó ooфol.

65 Cf. D. Dueck, in D. Dueck, H. Lindsay \& S. Pothecary (edd.), op. cit., p. 86-107, p. 96: Strabo quotes poetry when dealing with aspects of anthropology such as ethnical and physical characteristics, laws and habits of various foreigners and origins of nations.

66 Cf. N. Litinas, "Strabo's sources in the light of a tale", in D. Dueck, H. Lindsay \& S. Pothecary (edd.), op. cit., p. I07-117, p. 116: (...) The study of Strabo's sources for certain tales and myths may help us to 


\section{Conclusión}

Las reservas de Dumézil respecto a la herencia indoeuropea en la religión griega no impiden replantear la cuestión a la luz de los textos. Por otra parte, la relación de los pasajes de Calímaco con los cultos delios, esto es, con festividades que además congregaban a un público numeroso, matiza muy mucho la opinión de que dicha herencia se redujera al mero plano literario. Además, el horizonte cronológico que se extiende desde Arquíloco hasta Estrabón indica hasta qué punto los mitos antiguos permanecían en la cosmovisión de los griegos, a la que proporcionaban base y forma.

understand the circumstances under which he wrote. Strabo seems to have used material stored on the shelves of the Alexandrian library etc. 